\title{
Quantum-invariant processes in phase space
}

\author{
Alfredo Luis* \\ Departamento de Óptica, Facultad de Ciencias Físicas, Universidad Complutense, 28040 Madrid, Spain
}

(Received 6 June 2004; published 30 November 2004)

\begin{abstract}
We show the formal equivalence between the phase-space representations of transformations and quantum states. We study invariant quantum input-output transformations in phase space and in Hilbert space. We show that all invariant processes are linear transformations while the converse is not true. Some relevant examples of application of these ideas are examined.
\end{abstract}

DOI: $10.1103 /$ PhysRevA.70.052118

PACS number(s): 03.65.Ca, 42.50.-p

\section{INTRODUCTION}

Quantum physics admits phase-space formulations fully equivalent to the standard Hilbert formalism. States and observables are replaced by functions on classical phase space so that expected values are computed, as in classical statistical physics, by averaging over the phase space. This is valid for Cartesian and angular as well as finite-dimensional variables [1-7]. The classical-like appearance of phase-space approaches provides valuable physical insight and allows us to describe alike classical and quantum processes using one and the same language [8,9]. Moreover, the phase-space representatives can be determined experimentally, allowing direct observation of quantum states [1-7].

These techniques usually refer to quantum states, but recently it has been shown that there are also phase-space approaches for input-output transformations [10,11]. In this work we develop further this idea. More specifically, in Sec. II we demonstrate that the correspondence between transformations and phase-space distributions admits formally the same structure of standard phase-space correspondences for quantum states.

Furthermore, we focus on quantum-invariant processes in phase space. For these transformations the displacement of the input variables only produces a displacement of the output variables [12]. This idea is very easily embodied on the corresponding phase-space representative that must be just a function of the difference between the input and output variables. We study how this simple structure translates to the standard description of transformations in the Hilbert-space picture. More precisely, in Secs. III and IV we demonstrate two results: (i) all invariant processes are linear transformations, and (ii) the converse is not true and there are linear transformations that are not invariant. As a by-product we derive very general formulas for constructing the phasespace representative of invariant processes.

Linear transformations are very important since they describe very fundamental processes governed by quadratic Hamiltonians. They are easy to solve and are the basis for meaningful approximations to more complex systems. Furthermore, systems which are both linear and invariant are

*Electronic address: alluis@ fis.ucm.es; URL: http://www.ucm.es/ info/gioq particularly easy to analyze and encompass a great variety of phenomena in very different fields. Focusing on optics we can mention beam splitting, interference, imaging, and even field propagation in nonlinear media driven by strong pumps [12-15].

In Sec. II we recall the basis of the phase-space representation of transformations, focusing on the formal equivalence with the representation of quantum states. In Sec. III we define the invariant processes examining their relation with linear transformations. In Sec. IV we study some applications illustrating this formalism.

\section{PHASE-SPACE REPRESENTATION OF TRANSFORMATIONS}

In this section we briefly outline the phase-space description of the most general input-output transformation [11]. The input and output degrees of freedom of interest (the signal) will be represented by the Hilbert spaces $\mathcal{H}_{\text {in }}$ and $\mathcal{H}_{\text {out }}$, respectively. The corresponding quantum states are represented by the density matrices $\rho_{\text {in }}$ and $\rho_{\text {out }}$. In order to describe the most general transformation, including open as well as closed systems, we have to consider possible couplings of the system with additional degrees of freedom initially in a state $\rho_{\text {aux }}$ in a Hilbert space $\mathcal{H}_{\text {aux }}$. The Hilbert space $\mathcal{H}_{\text {aux }}$ is defined so that $\mathcal{H}_{\text {in }} \otimes \mathcal{H}_{\text {aux }}$ is a closed system and the input-output process is a unitary operator $U: \mathcal{H}_{\text {in }}$ $\otimes \mathcal{H}_{\text {aux }} \rightarrow \mathcal{H}_{\text {out }} \otimes \mathcal{H}_{\text {acc }}$, where $\mathcal{H}_{\text {acc }}$ is the Hilbert space needed to encompass the image of $\mathcal{H}_{\text {in }} \otimes \mathcal{H}_{\text {aux }}$.

We assume that the total input state factorizes $\rho_{\text {in }} \rho_{\text {aux }}$. The final density matrix for the signal $\rho_{\text {out }}$ in $\mathcal{H}_{\text {out }}$ arises after tracing over the variables $\mathcal{H}_{\text {acc }}$ :

$$
\rho_{\text {out }}=\operatorname{tr}_{\text {acc }}\left(U \rho_{\text {in }} \rho_{\text {aux }} U^{\dagger}\right)=\sum_{k} U_{k} \rho_{\text {in }} U_{k}^{\dagger},
$$

where the operators $U_{k}: \mathcal{H}_{\text {in }} \rightarrow \mathcal{H}_{\text {out }}$ verify

$$
\sum_{k} U_{k}^{\dagger} U_{k}=I_{\text {in }}
$$

$I_{\text {in }}$ being the identity in $\mathcal{H}_{\text {in }}$.

For definiteness, in what follows $\mathcal{H}_{\text {in }}$ and $\mathcal{H}_{\text {out }}$ represent unbounded, continuous, and a dimensionless Cartesian degrees of freedom $q_{j}, p_{j}, j=1, \ldots, n$ and $q_{k}^{\prime}, p_{k}^{\prime}, k=1, \ldots, n^{\prime}$, respectively. The corresponding operators satisfy the commutation relations 


$$
\left[\hat{q}_{j}, \hat{q}_{\ell}\right]=\left[\hat{p}_{j}, \hat{p}_{\ell}\right]=0, \quad\left[\hat{q}_{j}, \hat{p}_{\ell}\right]=i \delta_{j, \ell},
$$

and similarly for $\hat{q}_{k}^{\prime}, \hat{p}_{k}^{\prime}$. These variables can represent mechanical position and linear momentum, as well as the quadratures of electromagnetic field modes. To simplify the notation we will introduce the $2 n$ - and $2 n^{\prime}$-dimensional vectors, respectively,

$$
\mathbf{z}=\left(\begin{array}{c}
q_{1} \\
\vdots \\
q_{n} \\
p_{1} \\
\vdots \\
p_{n}
\end{array}\right), \quad \mathbf{z}^{\prime}=\left(\begin{array}{c}
q_{1}^{\prime} \\
\vdots \\
q_{n^{\prime}}^{\prime} \\
p_{1}^{\prime} \\
\vdots \\
p_{n^{\prime}}^{\prime}
\end{array}\right),
$$

and the associated quantum operators are denoted as $\hat{\mathbf{z}}, \hat{\mathbf{z}}^{\prime}$.

There are many complete formulations of the quantum theory on the classical phase space formed by Cartesian variables $[2,4,13]$. One of the most widely studied is the family of $\rho \leftrightarrow W$ correspondences between density matrices $\rho$ (or operators in general) and distributions $W$ on phase space labeled by a real parameter $s$ in the form $[4,13]$

$$
\begin{gathered}
W(\mathbf{z}, s)=\operatorname{tr}[\rho \Delta(\mathbf{z}, s)], \\
\rho=\frac{1}{(2 \pi)^{n}} \int d^{2 n} \mathbf{z} W(\mathbf{z}, s) \Delta(\mathbf{z},-s),
\end{gathered}
$$

where

$$
\Delta(\mathbf{z}, s)=\frac{1}{(2 \pi)^{n}} \int d^{2 n} \boldsymbol{y} e^{s y^{2} / 4} e^{i y^{T} K \mathbf{z}} e^{-i y^{T} K \hat{\mathbf{z}}}
$$

$\boldsymbol{y}$ is a $2 n$-dimensional real vector,

$$
K=\left(\begin{array}{cc}
0, & I_{n} \\
-I_{n}, & 0,
\end{array}\right)
$$

and $I_{n}$ is the $n \times n$ identity matrix. As particular cases we have the $Q$ function $(s=-1)$, the $P$ representation $(s=1)$, and the Wigner function $(s=0)[4,13]$. Note that the functions $W(\mathbf{z}, s)$ do not necessarily exist as ordinary functions for all values of $s$ and for all states.

Not only quantum states, but also processes can be fully described by functions on phase space $[8,9,11]$. The inputoutput transformation (2.1) can be expressed alternatively as a relation between distributions:

$$
W_{\text {out }}\left(\mathbf{z}^{\prime}, s^{\prime}\right)=\int d^{2 n} \mathbf{z} \mathcal{U}\left(\mathbf{z}, s ; \mathbf{z}^{\prime}, s^{\prime}\right) W_{\text {in }}(\mathbf{z}, s),
$$

where $W_{\text {in }}$ and $W_{\text {out }}$ are the distributions associated with $\rho_{\text {in }}$ and $\rho_{\text {out }}$, respectively, and

$$
\begin{aligned}
\mathcal{U}\left(\mathbf{z}, s ; \mathbf{z}^{\prime}, s^{\prime}\right) & =\frac{1}{(2 \pi)^{n}} \operatorname{tr}\left[\Delta\left(\mathbf{z}^{\prime}, s^{\prime}\right) U \Delta(\mathbf{z},-s) \rho_{\text {aux }} U^{\dagger}\right] \\
& =\frac{1}{(2 \pi)^{n}} \sum_{k} \operatorname{tr}_{\text {out }}\left[\Delta\left(\mathbf{z}^{\prime}, s^{\prime}\right) U_{k} \Delta(\mathbf{z},-s) U_{k}^{\dagger}\right] .
\end{aligned}
$$

Formally, $\mathcal{U}$ is the output corresponding to an impulse input $W_{\text {in }}(\mathbf{z}, s) \propto \delta^{(2 n)}\left(\mathbf{z}-\mathbf{z}_{0}\right)$.

We can show that the transformation-function correspondence can be expressed formally using the same relations valid for quantum states in Eq. (2.5). To this end we can use the Liouville formulation where operators are represented by vectors in a suitably doubled Hilbert space [16]:

$$
\left.\left.A=\sum_{n, m} A_{n m}|n\rangle\langle m|\leftrightarrow| A\rangle\right\rangle=\sum_{n, m} A_{n m}|n, m\rangle\right\rangle,
$$

where $|n\rangle$ is a orthonormal basis in the original Hilbert space and $|n, m\rangle\rangle$ is the associated basis in the enlarged space. This allows us to express the transformation-function correspondence in the form

$$
\begin{gathered}
\mathcal{U}\left(\mathbf{z}, s ; \mathbf{z}^{\prime}, s^{\prime}\right)=\operatorname{tr}\left[\rho_{\mathcal{U}} \widetilde{\Delta}\left(\mathbf{z}, s ; \mathbf{z}^{\prime}, s^{\prime}\right)\right], \\
\rho_{\mathcal{U}}=\frac{1}{(2 \pi)^{n^{\prime}-n}} \int d^{2 n} \mathbf{z} \int d^{2 n^{\prime}} \mathbf{z}^{\prime} \mathcal{U}\left(\mathbf{z}, s ; \mathbf{z}^{\prime}, s^{\prime}\right) \widetilde{\Delta}\left(\mathbf{z},-s ; \mathbf{z}^{\prime},-s^{\prime}\right),
\end{gathered}
$$

where

$$
\left.\rho_{\mathcal{U}}=\sum_{k}\left|U_{k}\right\rangle\right\rangle\left\langle\left\langle U_{k}\right|\right.
$$

and

$$
\begin{aligned}
\widetilde{\Delta}\left(\mathbf{z}, s ; \mathbf{z}^{\prime}, s^{\prime}\right)= & \frac{1}{(2 \pi)^{n}} \sum_{n, n^{\prime}, m, m^{\prime}}\left\langle n^{\prime}\left|\Delta\left(\mathbf{z}^{\prime}, s^{\prime}\right)\right| m^{\prime}\right\rangle\langle m|\Delta(\mathbf{z},-s)| n\rangle \\
& \left.\times\left|n^{\prime}, n\right\rangle\right\rangle\left\langle\left\langle m^{\prime}, m\right|\right. \\
= & \frac{1}{(2 \pi)^{n}} \Delta\left(\mathbf{z}^{\prime}, s^{\prime}\right) \otimes \Delta *(\mathbf{z},-s),
\end{aligned}
$$

where in the last equality it is understood that

$$
\left.\left|n^{\prime}, n\right\rangle\right\rangle\left\langle\left\langle m^{\prime}, m|=| n^{\prime}\right\rangle\left\langle m^{\prime}|\otimes| n\right\rangle\langle m|,\right.
$$

and the complex conjugation refers to the basis in Eq. (2.10). Note that $\rho_{\mathcal{U}}$ formally resembles a density matrix, $\rho_{\mathcal{U}}^{\dagger}=\rho_{\mathcal{U}}$ $\geqslant 0$, although $\operatorname{tr} \rho_{\mathcal{U}} \neq 1$.

Among other consequences of the above relations we have that, for two transformations $U$ and $V$,

$$
\begin{aligned}
\operatorname{tr}\left(\rho_{\mathcal{U}} \rho_{\mathcal{V}}\right)= & \frac{1}{(2 \pi)^{n^{\prime}-n}} \int d^{2 n} \mathbf{z} d^{2 n^{\prime}} \mathbf{z}^{\prime} \mathcal{U}_{U}\left(\mathbf{z}, s ; \mathbf{z}^{\prime}, s^{\prime}\right) \\
& \times \mathcal{U}_{V}\left(\mathbf{z},-s ; \mathbf{z}^{\prime},-s^{\prime}\right)=\left|\operatorname{tr}\left(U^{\dagger} V\right)\right|^{2},
\end{aligned}
$$

where the first equality is fully general while the last one is valid only for unitary transformations $U, V: \mathcal{H}_{\text {in }} \rightarrow \mathcal{H}_{\text {out }}$.

The input-output relation (2.1) can be also expressed as a relation between characteristic functions [13]: 


$$
\chi_{\text {out }}\left(\boldsymbol{\xi}^{\prime}, s^{\prime}\right)=\int d^{2 n} \tilde{\xi} \tilde{\mathcal{U}}\left(\boldsymbol{\xi}, s ; \boldsymbol{\xi}^{\prime}, s^{\prime}\right) \chi_{\text {in }}(\boldsymbol{\xi}, s),
$$

where $\chi_{\text {in }}$ and $\chi_{\text {out }}$ are the characteristic functions associated with $\rho_{\text {in }}$ and $\rho_{\text {out }}$, respectively,

$$
\chi(\boldsymbol{\xi}, s)=e^{s \xi^{2} / 4} \operatorname{tr}\left(\rho e^{-i \xi^{T} K \hat{\mathbf{z}}}\right),
$$

$\boldsymbol{\xi}$ and $\xi^{\prime}$ are real vectors of dimension $2 n$ and $2 n^{\prime}$, respectively, representing spatial frequencies in phase space, and

$$
\begin{aligned}
\tilde{\mathcal{U}}\left(\boldsymbol{\xi}, s ; \boldsymbol{\xi}^{\prime}, s^{\prime}\right)= & \frac{1}{(2 \pi)^{n}} e^{s^{\prime} \boldsymbol{\xi}^{\prime 2} / 4} e^{-s \xi^{2} / 4} \operatorname{tr}\left(e^{-i \boldsymbol{\xi}^{T \prime} K^{\prime} \hat{\mathbf{z}}^{\prime}} U e^{i \boldsymbol{\xi}^{T} K \hat{\mathbf{z}}} \rho_{\mathrm{aux}} U^{\dagger}\right) \\
= & \frac{1}{(2 \pi)^{n+n^{\prime}}} \int d^{2 n} \mathbf{z} \int d^{2 n^{\prime}} \mathbf{z}^{\prime} e^{-i \boldsymbol{\xi}^{T \prime} K^{\prime} \mathbf{z}^{\prime}} \\
& \times e^{i \boldsymbol{\xi}^{T} K \mathbf{z}} \mathcal{U}\left(\mathbf{z}, s ; \mathbf{z}^{\prime}, s^{\prime}\right),
\end{aligned}
$$

where $K^{\prime}$ is the matrix in Eq. (2.7) replacing $n$ by $n^{\prime}$. Characteristic functions and distributions are Fourier transform pairs, so both $W(\mathbf{z}, s)$ and $\chi(\xi, s)$ provide full information about the system state. Equivalently, the function $\tilde{\mathcal{U}}$ in Eqs. (2.16) and (2.18) is a Fourier transform of $\mathcal{U}$ and determines completely the transformation. The use of $\tilde{\mathcal{U}}$ or $\mathcal{U}$ is a matter of convenience and, in fact, many practical schemes measure characteristic functions instead of distributions [17]. In particular, phase-space tomography relies on measurement of the $s=0$ characteristic function [7]. The practical determination of phase-space representatives of transformations is studied in Ref. [11]. Moreover, characteristic functions always exist for every $\rho$ and $s$ even when the corresponding distribution $W(\mathbf{z}, s)$ does not exist. Most of the ensuing analysis is carried out using characteristic functions instead of distributions.

\section{QUANTUM-INVARIANT PROCESSES}

Invariant transformations (also referred to as shift invariant, stationary, or isoplanatic [12]) are defined to be of the form

$$
\mathcal{U}\left(\mathbf{z}^{\prime}, \mathbf{z}\right)=\mathcal{U}\left(\mathbf{z}^{\prime}-M \mathbf{z}\right)
$$

where $M$ is a $2 n^{\prime} \times 2 n$ constant matrix. The relation between input and output distributions is a convolution in phase space,

$$
W_{\text {out }}\left(\mathbf{z}^{\prime}\right)=\int d^{2 n} \mathbf{z} \mathcal{U}\left(\mathbf{z}^{\prime}-M \mathbf{z}\right) W_{\text {in }}(\mathbf{z})
$$

or a filtering in the frequency domain

$$
\chi_{\text {out }}\left(\boldsymbol{\xi}^{\prime}\right)=\tilde{\mathcal{U}}\left(\boldsymbol{\xi}^{\prime}\right) \chi_{\text {in }}\left(N \boldsymbol{\xi}^{\prime}\right)
$$

where $N=K M^{T} K^{\prime T}$, and $\tilde{\mathcal{U}}$ is the Fourier transform of $\mathcal{U}\left(z^{\prime}\right)$ in Eq. (3.1):

$$
\tilde{\mathcal{U}}\left(\boldsymbol{\xi}^{\prime}\right)=(2 \pi)^{n-n^{\prime}} \int d^{2 n^{\prime}} \mathbf{z}^{\prime} e^{-i \xi^{\prime T} K^{\prime} \mathbf{z}^{\prime}} \mathcal{U}\left(\mathbf{z}^{\prime}\right) .
$$

It is clear that the invariant character does not depend on the values of $s, s^{\prime}$. For simplicity we omit these parameters throughout.

The function $\tilde{\mathcal{U}}$ is the frequency response or transfer function (the analog of the optical transfer function in classical optical imaging). From Eq. (3.3) any invariant process can be regarded as a frequency filter modifying the phase-space spatial spectrum of the input state.

The experimental determination of invariant systems is rather simple. Since they formally depend only on one set of variables (the difference between input $\mathbf{z}$ and output $\mathbf{z}^{\prime}$ variables), it is sufficient to determine the output state for a single input state (the vacuum, for instance)[11].

As far as we refer to the phase-space picture, the above definition applies to the classical as well as to the quantum domains alike. In order to fully develop these ideas in the quantum domain it is necessary to find out which quantum processes are actually invariant in phase space and how are represented in the Hilbert-space picture. This point is addressed next.

In principle, the very same definition of phase-space invariance suggests that they might be related to linear transformations. In this sense previous results show that linear unitary transformations between closed systems are actually invariant. In particular, $\mathcal{U}$ becomes a $\delta$ function for $s=s^{\prime}=0$ $[8,9]$. In what follows we examine the equivalence between linear and invariant processes in full generality, including open systems and even output Hilbert spaces not unitarily equivalent to the input one (i.e., $\mathcal{H}_{\text {in }} \neq \mathcal{H}_{\text {out }}$ ) because of different dimensionality $n \neq n^{\prime}$ for example. We will show that the invariant processes are a subset of the linear input-output transformations

$$
U^{\dagger} \hat{\mathbf{z}}^{\prime} U=\hat{M} \hat{\mathbf{z}}+\hat{\boldsymbol{V}}
$$

where $U$ is the global unitary operator, $\hat{M}$ is a $2 n^{\prime} \times 2 n$ matrix, and $\hat{V}$ is a $2 n^{\prime}$-dimensional vector. Both $\hat{M}$ and $\hat{V}$ are independent of $\hat{\mathbf{z}}$, but they can be still operators acting on $\mathcal{H}_{\text {aux }}$.

In most cases the unitary representation $U$ in the extended space $\mathcal{H}_{\text {in }} \otimes \mathcal{H}_{\text {aux }}$ is not available, so it would be desirable to deal with another characterization of linear transformations equivalent to Eq. (3.5). For transformations of the form (3.5) the mean value of the product of $k^{\prime}$ output operators will be a linear function of the mean value of products of $k$ input operators with $k \leqslant k^{\prime}$,

$$
\left\langle\hat{z}_{j_{1}}^{\prime} \cdots \hat{z}_{j_{k^{\prime}}}^{\prime}\right\rangle=\sum_{k=1}^{k^{\prime}} \sum_{\ell_{1}, \ldots, \ell_{k}=1}^{2 n} M_{j_{1}, \ldots, j_{k^{\prime}}}^{\ell_{1}, \ldots, \ell_{k}}\left\langle\hat{z}_{\ell_{1}} \cdots \hat{z}_{\ell_{k}}\right\rangle+V_{j_{1}, \ldots, j_{k^{\prime}}},
$$

for some constant coefficients $M_{j_{1}, \ldots, j_{k^{\prime}}}^{\ell_{1}, \ldots, \ell_{j_{1}}, \ldots, j_{k^{\prime}}}$ depending only on $\hat{M}, \hat{\mathbf{V}}$, and $\rho_{\text {aux }}$. This generalized expression is a suitable alternative definition particularly useful for our purposes. 
Next we demonstrate that every invariant quantum process is a linear input output transformation. We consider first the case $k^{\prime}=1$. From Eq. (2.16),

$$
\left\langle\hat{z}_{k}^{\prime}\right\rangle=\left.i \sum_{j=1}^{2 n^{\prime}} K_{j, k}^{\prime} \frac{\partial \chi_{\text {out }}\left(\boldsymbol{\xi}^{\prime}\right)}{\partial \xi_{j}^{\prime}}\right|_{\xi^{\prime}=0},
$$

while from Eq. (3.3)

$$
\left.\frac{\partial \chi_{\text {out }}\left(\boldsymbol{\xi}^{\prime}\right)}{\partial \xi_{j}^{\prime}}\right|_{\xi^{\prime}=0}=\left.\frac{\partial \tilde{\mathcal{U}}\left(\xi^{\prime}\right)}{\partial \xi_{j}^{\prime}}\right|_{\xi^{\prime}=0}+\left.\frac{\partial \chi_{\text {in }}\left(N \boldsymbol{\xi}^{\prime}\right)}{\partial \xi_{j}^{\prime}}\right|_{\xi^{\prime}=0},
$$

and we have used that $\tilde{\mathcal{U}}(0)=\chi(0)=1$. The right-hand side of Eq. (3.8) can be expressed using Eqs. (3.4) and (2.16) as

$$
\begin{gathered}
\left.\frac{\partial \tilde{\mathcal{U}}\left(\boldsymbol{\xi}^{\prime}\right)}{\partial \xi_{j}^{\prime}}\right|_{\xi^{\prime}=0}=-i(2 \pi)^{\left(n-n^{\prime}\right)} \sum_{\ell=1}^{n^{\prime}} K_{j, \ell}^{\prime} \int d^{2 n^{\prime}} \mathbf{z}^{\prime} z_{\ell}^{\prime} \mathcal{U}\left(\mathbf{z}^{\prime}\right), \\
\left.\frac{\partial \chi_{\text {in }}\left(N \boldsymbol{\xi}^{\prime}\right)}{\partial \xi_{j}^{\prime}}\right|_{\xi^{\prime}=0}=-i \sum_{\ell, m=1}^{n} N_{\ell, j} K_{\ell, m}\left\langle\mathbf{z}_{m}\right\rangle,
\end{gathered}
$$

where the matrix $N$ has been defined after Eq. (3.3). From the above equations we finally get

$$
\left\langle\hat{\mathbf{z}}^{\prime}\right\rangle=M\langle\hat{\mathbf{z}}\rangle+\boldsymbol{V},
$$

where

$$
\mathbf{V}=(2 \pi)^{\left(n-n^{\prime}\right)} \int d^{2 n^{\prime}} \mathbf{z}^{\prime} \mathcal{U}\left(\mathbf{z}^{\prime}\right) \mathbf{z}^{\prime}
$$

Thus we have obtained that for invariant systems the input-output relation is clearly of the form (3.6) for $k^{\prime}=1$. Following this same procedure we can see that this is also always valid for higher orders $k^{\prime}>1$. Therefore, invariant systems are linear transformations.

However, the converse is not true, and not all linear transformations are invariant processes. This can be seen by examining the output characteristic function when the inputoutput transformation is of the form (3.5):

$$
\chi_{\text {out }}\left(\boldsymbol{\xi}^{\prime}\right)=e^{s^{\prime} \xi^{\prime 2} / 4} \operatorname{tr}\left[\rho_{\text {in }} \rho_{\text {aux }} e^{-i\left(\xi^{\prime T} K^{\prime} \hat{M} \hat{\mathbf{z}}+\xi^{\prime T} K^{\prime} \hat{\mathbf{V}}\right)}\right] .
$$

In the most general case $\hat{M}$ and $\hat{\mathbf{V}}$ are operators acting on $\mathcal{H}_{\text {aux }}$ and the right-hand side of the above equation does not factorize in the form (3.3) required for invariance. A particular simple example is presented in Sec. IV. Nevertheless, there are many important situations where the right-hand side factorizes, as demonstrated also by some examples in the next section.

\section{EXAMPLES}

In this section we present some examples illustrating the results of the preceding sections by applying this formalism to some linear (invariant and noninvariant) quantum processes. As we have mentioned above, linear transformations (the only candidates to be invariant process) are very com- mon in quantum physics. Throughout we will focus on a single degree of freedom describable by an annihilation operator $a=(q+i p) / \sqrt{2}$ as the only input and output variable $\mathcal{H}_{\text {in }}=\mathcal{H}_{\text {out }}$. This can be representing the one-dimensional motion of a material system or the complex amplitude of a single mode of the electromagnetic field.

\section{A. Linear transformations with constant coefficients}

Maybe the most frequent situation arising in real physical processes corresponds to the case of transformations of the form (3.5) with constant coefficients and linear in the auxiliary variables

$$
U^{\dagger} \hat{\mathbf{z}}^{\prime} U=M \hat{\mathbf{z}}+V \hat{\tilde{\mathbf{z}}},
$$

where $M$ is a $2 n^{\prime} \times 2 n$ constant matrix, $V$ is a $2 n^{\prime} \times 2 m$ constant matrix, the $2 m$-dimensional vector $\hat{\tilde{\mathbf{z}}}$ is made of $m$ position $\widetilde{q}_{j}$ operators, and $m$ momentum $\tilde{p}_{j}$ operators $j$ $=1, \ldots, m$, as in Eq. (2.4) acting on the auxiliary system space with commutation relations $\left[\hat{\tilde{z}}_{j}, \hat{\tilde{z}}_{\ell}\right]=i \widetilde{K}_{j, \ell}$, where $\tilde{K}$ is given by Eq. (2.7) after replacing $I_{n}$ by $I_{m}$. The preservation of the commutation relations imposes

$$
M K M^{T}+V \tilde{K} V^{T}=K^{\prime} .
$$

From Eq. (3.12) we have that the transformation is of the form (3.3) with

$$
\tilde{\mathcal{U}}\left(\xi^{\prime}\right)=e^{s^{\prime} \xi^{2} / 4} e^{-s \xi^{\prime T} N^{T} N \xi^{\prime} / 4} \operatorname{tr}\left(\rho_{\text {aux }} e^{-i \xi^{T} K^{\prime} V \hat{\tilde{z}}}\right),
$$

where it can be appreciated that $\tilde{\mathcal{U}}$ is proportional to the characteristic function of the state of the auxiliary system,

$$
\tilde{\mathcal{U}}\left(\boldsymbol{\xi}^{\prime}\right)=e^{-\xi^{\prime T} G \xi^{\prime} / 2} \chi_{\text {aux }}(\boldsymbol{\eta}),
$$

where $G$ is a symmetric $2 n^{\prime} \times 2 n^{\prime}$ constant matrix,

$$
G=\frac{1}{2} K^{\prime}\left(\widetilde{s} V V^{T}+s M M^{T}-s^{\prime}\right) K^{\prime T},
$$

$\widetilde{s}$ is the $s$ value for the characteristic function $\chi_{\text {aux }}$, and

$$
\boldsymbol{\eta}=\tilde{K} V^{T} K^{\prime T} \boldsymbol{\xi}^{\prime} .
$$

This implies that the phase-space representative of the transformation $\mathcal{U}\left(\mathbf{z}^{\prime}\right)$ is proportional to a Gaussian convolution of the phase-space representative of $\rho_{\text {aux }}$,

$$
\mathcal{U}\left(\mathbf{z}^{\prime}\right)=(2 \pi)^{n^{\prime}-n-m} \int d^{2 m} \widetilde{\mathbf{z}} H\left(\mathbf{z}^{\prime}-V \widetilde{\mathbf{z}}\right) W_{\text {aux }}(\widetilde{\mathbf{z}}),
$$

where

$$
\begin{aligned}
H\left(\mathbf{z}^{\prime}\right) & =\frac{1}{(2 \pi)^{2 n^{\prime}}} \int d^{2 n^{\prime}} \boldsymbol{\xi}^{\prime} e^{i \boldsymbol{\xi}^{\prime T} K^{\prime} \mathbf{z}^{\prime}} e^{-\xi^{\prime T} G \xi^{\prime} / 2} \\
& =\frac{1}{\sqrt{(2 \pi)^{2 n^{\prime}} \operatorname{det} G}} e^{-\mathbf{z}^{\prime T} G^{-1} \mathbf{z}^{\prime} / 2}
\end{aligned}
$$

provided that the corresponding integrals can be performed.

These expressions are valid for arbitrary transformations with constant coefficients. For instance this includes losses and amplification as we shall show below. 
The above expressions simplify for the usual case of phase-insensitive transformations [18]. These are transformations for which the output annihilation operators (proportional to $\left.q_{j}^{\prime}+i p_{j}^{\prime}\right)$ are proportional to just annihilation operators for the input (proportional to $q_{j}+i p_{j}$ and $\widetilde{q}_{j}+i \widetilde{p}_{j}$ ). This implies further constraints on $M$ and $V$. More specifically, if we express these matrices in terms of the submatrices acting on the position and momentum variables,

$$
M=\left(\begin{array}{ll}
M_{1} & M_{2} \\
M_{3} & M_{4}
\end{array}\right), \quad V=\left(\begin{array}{ll}
V_{1} & V_{2} \\
V_{3} & V_{4}
\end{array}\right),
$$

we get that for phase-insensitive transformations $M_{4}=M_{1}$, $M_{3}=-M_{2}$ and $V_{4}=V_{1}, V_{3}=-V_{2}$. Among other consequences this implies

$$
M K M^{T}=K^{\prime} M M^{T}, \quad V \tilde{K} V^{T}=K^{\prime} V V^{T},
$$

so that relation (4.2) becomes

$$
M M^{T}+V V^{T}=I_{2 n^{\prime}} .
$$

This simplifies notably the expression for $G$ when $\widetilde{s}=s^{\prime}=s$, since in such a case $G=0$ and then we have

$$
\tilde{\mathcal{U}}\left(\boldsymbol{\xi}^{\prime}\right)=\chi_{\text {aux }}\left(\widetilde{K} V^{T} K^{\prime T} \boldsymbol{\xi}^{\prime}\right)
$$

and

$$
\mathcal{U}\left(\mathbf{z}^{\prime}\right)=(2 \pi)^{n^{\prime}-n-m} \int d^{2 m} \widetilde{\mathbf{z}} \delta\left(\mathbf{z}^{\prime}-V \widetilde{\mathbf{z}}\right) W_{\text {aux }}(\widetilde{\mathbf{z}}) .
$$

For the particular but frequent case that the matrix $V$ is square and invertible we get

$$
\mathcal{U}\left(\mathbf{z}^{\prime}\right)=\frac{(2 \pi)^{n^{\prime}-n-m}}{|\operatorname{det} V|} W_{\text {aux }}\left(V^{-1} \mathbf{z}^{\prime}\right) .
$$

This last expression agrees with recent results obtained when studying real physical situations [14,15]. In Ref. [14] the quantum transformation performed by dispersive and absorbing four-port devices (such as real beam splitters) is constructed. In such a case the variables $z, z^{\prime}$ represent the twomode input and output fields, respectively, while $\tilde{z}$ represent the inner state of the transforming device. When the transformation is expressed in the phase-space picture [Eq. (82) in Ref. [14]] the expression obtained is of the form (3.2) with $\mathcal{U}\left(\mathbf{z}^{\prime}\right)$ given by Eq. (4.14). Another example that fits this model studies the quantum-state extraction from high- $Q$ cavities [15]. In this example the variables $z$ represent the field inside a cavity, and $z^{\prime}$ represent the field leaving the cavity while $\tilde{z}$ represent the field incident on the cavity as well as any other variables required to account for absorption. Also in this case the relation between the field inside and outside the cavity in the phase-space picture [Eq. (54) in Ref. [15]] is of the form (3.2) with $\mathcal{U}\left(\mathbf{z}^{\prime}\right)$ given by Eq. (4.14).

\section{B. Amplification}

Classically an amplifier transforms an input phase space point $\alpha$ (i.e., the complex amplitude of an electromagnetic field mode) in the output one $\alpha^{\prime}=\mu \alpha$, where $\mu$ is constant
$[13,18]$. In quantum theory there is no input-output transformation $a^{\prime}=\mu a$ since commutations relations would not be preserved. Then, the quantum phase-insensitive amplifier must be an open system implicating auxiliary degrees of freedom $\mathcal{H}_{\text {aux }}$. In the most simple realization the signal to be amplified is represented by a ladder operator $a$ acting on $\mathcal{H}_{\text {in }}=\mathcal{H}_{\text {out }}$, while the auxiliary system is represented by a ladder operator $b$ acting on $\mathcal{H}_{\text {aux }}=\mathcal{H}_{\text {acc }}$. The coupling is governed by the interaction Hamiltonian $H=\hbar \kappa\left(a^{\dagger} b^{\dagger}+a b\right)$ where $\kappa$ is a coupling constant. In the case of field modes this interaction occurs in nonlinear crystals [13], while in the case of ion traps it can be implemented by using the methods in Ref. [19]. After a time $\tau$ the unitary operator representing the interaction in the total Hilbert space $\mathcal{H}_{\text {in }} \otimes \mathcal{H}_{\text {aux }}$ is $U=\exp \left[-i \kappa \tau\left(a^{\dagger} b^{\dagger}+a b\right)\right]$, leading to $a^{\prime}=U^{\dagger} a U=\mu a+\nu b^{\dagger}$, where $\mu=\cosh (\kappa \tau)$ and $\nu=-i \sinh (\kappa \tau)$. While classically it would be possible to fix the initial conditions such that $b$ $=0$, this is not possible in the quantum domain where $b$ is a quantum fluctuating variable. Its effects can be minimized by a proper choice of the initial state in $\mathcal{H}_{\text {aux }}$ such as $\rho_{\text {aux }}$ $=|0\rangle\langle 0|$ where $|0\rangle$ is the vacuum $b|0\rangle=0$. This leads to

$$
\rho_{\text {out }}=\frac{1}{\mu^{2}} \mu^{-a^{\dagger} a} \sum_{k=0}^{\infty} \frac{|\nu|^{2 k}}{k !} a^{\dagger k} \rho_{\text {in }} a^{k} \mu^{-a^{\dagger} a} .
$$

The phase-space representation of this transformation can be easily obtained by computing the $Q$ function for the output field $Q=\left\langle\alpha\left|\rho_{\text {out }}\right| \alpha\right\rangle$, where $|\alpha\rangle$ are coherent states, so that

$$
W_{\text {out }}\left(\mathbf{z}^{\prime}, s^{\prime}=-1\right)=\frac{1}{\mu^{2}} W_{\text {in }}\left(\frac{\mathbf{z}^{\prime}}{\mu}, s=-1\right) .
$$

In the frequency domain this leads to an input-output relation of the form (3.3) with

$$
\tilde{\mathcal{U}}\left(\boldsymbol{\xi}^{\prime}\right)=e^{\left(s^{\prime}+1-\mu^{2} s-\mu^{2}\right) \xi^{\prime 2} / 4}
$$

and $N=\mu$.

\section{Losses}

Losses are often conveniently described by the master equation (for a reservoir at zero temperature)

$$
\dot{\rho}=-\kappa\left(a^{\dagger} a \rho+\rho a^{\dagger} a-2 a \rho a^{\dagger}\right),
$$

which after an interaction time $\tau$ leads to the input-output relation

$$
\rho_{\text {out }}=\mu^{a^{\dagger} a} \sum_{n=0}^{\infty} \frac{\left(1-\mu^{2}\right)^{n}}{n !} a^{n} \rho_{\text {in }} a^{\dagger n} \mu^{a^{\dagger} a},
$$

where $\mu=e^{-\kappa \tau}$. The phase-space representative of this transformation can be easily computed by using the $P$ representation $(s=1)$ for $\rho_{\text {in }}$ :

$$
\rho_{\text {in }}=\frac{1}{\pi} \int d^{2} \alpha P_{\text {in }}(\alpha)|\alpha\rangle\langle\alpha|,
$$

where $|\alpha\rangle$ are the coherent states $a|\alpha\rangle=\alpha|\alpha\rangle$. With the help of this representation it is easy to arrive at 


$$
W_{\text {out }}\left(\mathbf{z}^{\prime}, s^{\prime}=1\right)=\frac{1}{\mu^{2}} W_{\text {in }}\left(\frac{\mathbf{z}^{\prime}}{\mu}, s=1\right) .
$$

In the frequency domain this leads to an input-output relation of the form (3.3) with

$$
\tilde{\mathcal{U}}\left(\boldsymbol{\xi}^{\prime}\right)=e^{\left(s^{\prime}-1-\mu^{2} s+\mu^{2}\right) \xi^{2} / 4}
$$

and $N=\mu$. We can appreciate a formal similarity between amplification and losses.

\section{Linear noninvariant transformation}

The next example shows that the invariant character of the transformation can rely upon the particular initial state of the auxiliary degrees of freedom and not only on the nature of the coupling. This is the case of an interaction governed by the Hamiltonian

$$
H=\hbar \kappa a^{\dagger} a s_{z},
$$

where $s_{z}=|+\rangle\langle+|-|-\rangle\langle-|$ and $| \pm\rangle$ are two orthogonal states of a two-dimensional auxiliary system $\mathcal{H}_{\text {aux }}=\mathcal{H}_{\text {acc }}$. The inputoutput transformation after an interaction time $\tau$ is always a linear transformation of the form (3.5) for the signal variables

$$
U^{\dagger} a U=e^{-i \phi s_{z}} a
$$

where $\phi=\kappa \tau$. The output characteristic function is

$$
\chi_{\text {out }}\left(\boldsymbol{\xi}^{\prime}\right)=e^{\left(s^{\prime}-s\right) \xi^{\prime 2} / 4}\left[p_{+} \chi_{\text {in }}\left(R^{T} \boldsymbol{\xi}^{\prime}\right)+p_{-} \chi_{\text {in }}\left(R \boldsymbol{\xi}^{\prime}\right)\right],
$$

where

$$
R=\left(\begin{array}{cc}
\cos \phi, & \sin \phi \\
-\sin \phi & \cos \phi
\end{array}\right)
$$

and $p_{ \pm}=\left\langle \pm\left|\rho_{\text {aux }}\right| \pm\right\rangle$ are the probabilities that the auxiliary system is initially in $| \pm\rangle$.

If $p_{+} p_{-}=0$ or $\sin \phi=0$, the transformation is clearly invariant. On the other hand, if $p_{+} p_{-} \neq 0$ and $\sin \phi \neq 0$, the expression (4.25) is not of the form (3.3) and the transformation is not invariant.

\section{CONCLUSIONS}

We have shown the formal equivalence between the phase-space representation of quantum states and transformations. We have introduced and studied the main properties of the invariant quantum input-output transformations. We have demonstrated that all invariant processes are linear while the converse is not true. Some relevant examples of application of these ideas have been examined.
[1] U. Leonhardt and H. Paul, Prog. Quantum Electron. 19, 89 (1995); U. Leonhardt, Measuring the Quantum State of Light (Cambridge University Press, Cambridge, England, 1997); D.-G. Welsch, W. Vogel, and T. Opatrný, in Progress in Optics, edited by E. Wolf (Elsevier Science, Amsterdam, 1999), Vol. 39.

[2] G. S. Agarwal and E. Wolf, Phys. Lett. 26A, 485 (1968); Phys. Rev. Lett. 21, 180 (1968); G. S. Agarwal, Phys. Rev. 177, 400 (1969); G. S. Agarwal and E. Wolf, Phys. Rev. D 2, 2161 (1970); 2, 2187 (1970); 2, 2206 (1970).

[3] C. H. Page, J. Appl. Phys. 23, 103 (1952); H. Margenau and R. N. Hill, Prog. Theor. Phys. 26, 722 (1961); C. L. Mehta, J. Math. Phys. 5, 677 (1964); L. Cohen, ibid. 7, 781 (1966); N. C. Dias and J. N. Prata, Phys. Lett. A 302, 261 (2002); R. Gase, T. Gase, and K. Blüthner, Opt. Lett. 20, 2045 (1995).

[4] K. E. Cahill and R. J. Glauber, Phys. Rev. 177, 1857 (1969); 177, 1882 (1969); J. Peřina, Coherence of Light (Reidel, Dordrecht, 1985).

[5] D. M. Kaplan and G. C. Summerfield, Phys. Rev. 187, 639 (1969); F. T. Arecchi, E. Courtens, R. Gilmore, and H. Thomas, Phys. Rev. A 6, 2211 (1972); N. Mukunda, Am. J. Phys. 47, 182 (1979); J. P. Bizarro, Phys. Rev. A 49, 3255 (1994); J. Vaccaro, ibid. 52, 3474 (1995); Opt. Commun. 113, 421 (1995); G. S. Agarwal, Phys. Rev. A 24, 2889 (1981); M. O. Scully, Phys. Rev. D 28, 2477 (1983); J. C. Várilly and J. M. Gracia-Bondía, Ann. Phys. (N.Y.) 190, 107 (1989); A. Luis and L. L. Sánchez-Soto, Quantum Opt. 5, 33 (1993); G. Ramachandran, A. R. Usha-Devi, P. Devi, and S. Sirsi, Found. Phys. 26, 401 (1996); V. V. Dodonov and V. I. Man'ko, Phys.
Lett. A 229, 335 (1997); C. Brif and A. Mann, Phys. Rev. A 59, 971 (1999); S. M. Chumakov, A. Frank, and K. B. Wolf, ibid. 60, 1817 (1999); S. M. Chumakov, A. B. Klimov, and K. B. Wolf, ibid. 61, 034101 (2000); V. P. Karassiov and A. V. Masalov, J. Opt. B: Quantum Semiclassical Opt. 4, S366 (2002).

[6] W. K. Wootters, Ann. Phys. (N.Y.) 176, 1 (1987); O. Cohendet, Ph. Combe, M. Sirugue, and M. Sirugue-Collin, J. Phys. A 21, 2875 (1988); D. Galetti, and A. F. R. Toledo Piza, Physica A 149, 267 (1988); J. A. Vaccaro and D. T. Pegg, Phys. Rev. A 41, 5156 (1990); A. Lukš and V. Peřinová, Quantum Opt. 6, 125 (1994); P. Kasperkovitz and M. Peev, Ann. Phys. (N.Y.) 230, 21 (1994); U. Leonhardt, Phys. Rev. Lett. 74, 4101 (1995); Phys. Rev. A 53, 2998 (1996); T. Opatrný, D.-G. Welsch, and B. Bužek, ibid. 53, 3822 (1996); A. Luis and J. Peřina, J. Phys. A 31, 1423 (1998); C. Miquel, J. P. Paz, and M. Saraceno, ibid. 65, 062309 (2002); J. P. Paz, ibid. 65, 062311 (2002).

[7] K. Vogel and H. Risken, Phys. Rev. A 40, 2847 (1989); D. T. Smithey, M. Beck, M. G. Raymer, and A. Faridani, Phys. Rev. Lett. 70, 1244 (1993); S. Schiller, G. Breitenbach, S. F. Pereira, T. Müller, and J. Mlynek, ibid. 77, 2933 (1996); G. Breitenbach, S. Schiller, and J. Mlynek, Nature (London) 387, 471 (1997).

[8] G. García Calderón and M. Moshinsky, J. Phys. A 13, L185 (1980); R. Dirl, P. Kasperkovitz, and M. Moshinsky, ibid. 21, 1835 (1988); A. K. Ekert and P. L. Knight, Phys. Rev. A 43, 3934 (1991).

[9] A. Walther, J. Opt. Soc. Am. 58, 1256 (1968); M. J. Bastiaans, 
Opt. Commun. 25, 26 (1978); 30, 321 (1979); J. Opt. Soc. Am. A 3, 1227 (1986); R. Simon, E. C. G. Sudarshan, and N. Mukunda, Phys. Rev. A 29, 3273 (1984); D. Dragoman, in Progress in Optics, edited by E. Wolf (North-Holland, Amsterdam, 1997), Vol. 37, p. 1; G. S. Agarwal, J. T. Foley, and E. Wolf, Opt. Commun. 62, 67 (1987); R. Simon, N. Mukunda, and E. C. G. Sudarshan, ibid. 65, 322 (1988); special issue on Wigner function in optics [J. Opt. Soc. Am. A 17(12), 2274 (2000)].

[10] J. F. Poyatos, J. I. Cirac, and P. Zoller, Phys. Rev. Lett. 78, 390 (1997); I. L. Chuang and M. A. Nielsen, J. Mod. Opt. 44, 2455 (1997); G. M. D'Ariano and L. Maccone, Phys. Rev. Lett. 80, 5465 (1998); V. Bužek, Phys. Rev. A 58, 1723 (1998); R. Gutzeit, S. Wallentowitz, and W. Vogel, ibid. 61, 062105 (2000); A. M. Childs, I. L. Chuang, and D. W. Leung, ibid. 64, 012314 (2001); J. Fiurášek and Z. Hradil, ibid. 63, 020101(R) (2001); J. Fiurášek, ibid. 64, 024102 (2001); G. M. D’Ariano and P. Lo Presti, Phys. Rev. Lett. 86, 4195 (2001); e-print quant-ph/0211133; F. De Martini, A. Mazzei, M. Ricci, and G. D’Ariano, e-print quant-ph/0210210; J. B. Altepeter, D. Branning, E. Jeffrey, T. C. Wei, P. G. Kwiat, R. T. Thew, J. L. O'Brien, M. A. Nielsen, and A. G. White, Phys. Rev. Lett. 90, 193601 (2003).
[11] A. Luis and L. L. Sánchez-Soto, Phys. Lett. A 261, 12 (1999); Phys. Rev. Lett. 83, 3573 (1999); A. Luis, Phys. Rev. A 62, 054302 (2000).

[12] J. D. Gaskill, Linear Systems, Fourier Transforms and Optics (Wiley, New York, 1978); J. W. Goodman, Introduction to Fourier Optics (McGraw-Hill, New York, 1996).

[13] J. Peřina, Quantum Statistics of Linear and Nonlinear Optical Phenomena, 2nd ed. (Kluwer Academic, Dordrecht, 1991).

[14] L. Knöll, S. Scheel, E. Schmidt, D.-G. Welsch, and A. V. Chizhov, Phys. Rev. A 59, 4716 (1999).

[15] M. Khanbekyan, L. Knöll, A. A. Semenov, W. Vogel, and D.-G. Welsch, Phys. Rev. A 69, 043807 (2004).

[16] U. Fano, Rev. Mod. Phys. 29, 74 (1957); A. Royer, Phys. Rev. A 43, 44 (1991).

[17] M. Wilkens and P. Meystre, Phys. Rev. A 43, 3832 (1991); S. M. Dutra P. L. Knight, and H. Moya-Cessa, ibid. 48, 3168 (1993); S. M. Dutra and P. L. Knight, ibid. 49, 1506 (1994); M. S. Kim, G. Antesberger, C. T. Bodendorf, and H. Walther, ibid. 58, R65 (1998); P. J. Bardroff, M. T. Fontenelle, and S. Stenholm, ibid. 59, R950 (1999); M. S. Kim and G. S. Agarwal, ibid. 59, 3044 (1999).

[18] C. M. Caves, Phys. Rev. D 26, 1817 (1982).

[19] S. Wallentowitz and W. Vogel, Phys. Rev. A 55, 4438 (1997). 